\title{
Features the Self-Diffusion of Oil through Porous Media of Clay
}

\author{
Nariman Kamilovich Dvoyashkin \\ Faculty of Oil and Gas, Almetyevsk State Oil Institute, Almetyevsk 423450, Tatarstan, Russia
}

\begin{abstract}
Absract: Self-diffusion features of natural hydrocarbon liquids through porous medium of clay have been studied by PFG NMR method. The samples of oil fields in the Republic of Tatarstan (Russia) were investigated. These were samples of ordinary (light) and highly viscous (heavy) crude oil. As heavy oil was taken the samples of residual oil and high-temperature fraction were extracted from conventional oil. Fluids have been studied both in pure (bulk) form as well as enclosed in porous medium. As the porous medium was used kaolinite (clay mineral) with a specific surface area $S_{1} \approx 9 \mathrm{~m}^{2} / \mathrm{g}$. The dependencies of the effective self-diffusion coefficients $D_{\text {eff }}$ on the mass fraction $\omega_{1}$ of fluid in a medium of kaolinite were investigated. It was found that for systems the heavy oil - kaolinite was observed the usual dependencies $D_{\text {eff }}\left(\omega_{1}\right)$, which indicatesthat the liquid's molecular mobility is decreased with increase of share obstacles in the samples. An anomalously enhanced self-diffusion in the samples of light oil - kaolinite was detected under certain conditions. These conditions are partial filling of porous space the kaolinite by liquid and a sufficiently high temperature in the system. The unusual state of enhanced self-diffusion is caused by the presence of the vapor liquid in the free part of pore space. A fast molecular exchange between coexisting the liquid and gas phases in the samples light oil - kaolinite provides realization the enhanced self-diffusion in them.
\end{abstract}

Key words: Self-diffusion, NMR PFG method, oil, porous media, gas-like state .

\section{Nomenclature}

$\begin{array}{ll}L O & \text { Light oil } \\ H O & \text { Heavy oil } \\ S_{1} & \text { Specific surface area } \\ \omega_{1} & \text { Mass fractions } \\ S D & \text { Self-diffusion } \\ P F G N M R & \text { Pulse field gradient nuclear magnetic resonance } \\ T & \text { Temperature } \\ R F & \text { Radiofrequency } \\ g & \text { Intensity of field gradient pulses } \\ t_{d} & \text { Observation time the diffusion process } \\ S D C & \text { Self-diffusion coefficient } \\ T_{1} & \text { Longitudinal relaxation time } \\ T_{2} & \text { Transverse relaxation time } \\ A_{s t i m} & \text { Stimulated spin echo amplitude initial } \\ A_{0} & \text { amplitude of the free induction signal } \\ & \text { After applying the first } 90^{\circ} \text { - radiofrequency } \\ D A & \text { (RF) pulse } \\ D & \text { Diffusion attenuation of the spin echo } \\ & \text { amplitude Owing to the self-diffusion process } \\ & \text { Self-diffusion coefficient }\end{array}$

Corresponding author: Nariman Kamilovich Dvoyashkin, Ph.D., professor, research fields: the physics of molecular systems and NMR of liquids in the porous medium.

\begin{tabular}{|c|c|}
\hline$D_{\text {eff }}$ & $\begin{array}{l}\text { Effective or average for the entire sample } \\
\text { volume } S D C\end{array}$ \\
\hline$P(D)$ & $\begin{array}{l}\text { Share of the resonating nuclei in a sample with } \\
\text { a value for } S D C \text { in interval from } D \text { to } \\
(D+d D)\end{array}$ \\
\hline$<r^{2}>$ & $\begin{array}{l}\text { Mean-square values of runsof diffusing } \\
\text { molecules }\end{array}$ \\
\hline$<d>$ & Average pore size \\
\hline$V_{0}$ & Specific pore volume \\
\hline$V_{T}$ & Total sample specific volume \\
\hline$V$ & Specific volume of liquid in a sample \\
\hline$D_{0}$ & $\begin{array}{l}\text { Self-diffusion coefficient for the pure (bulk) } \\
\text { liquid }\end{array}$ \\
\hline$t^{L}$ & "Lifetime" of the molecule in the liquid phase \\
\hline$t^{G}$ & "Lifetime" of the molecule in the gas phase \\
\hline$D^{L}$ & $S D C$ value in a typical liquid state \\
\hline$D^{G}$ & $S D C$ value in a typical gaseous state \\
\hline$P^{L}$ & $\begin{array}{l}\text { Relative fraction of molecules in a typical liquid } \\
\text { state }\end{array}$ \\
\hline$P^{G}$ & $\begin{array}{l}\text { Relative fraction of molecules in a typical } \\
\text { gaseous state }\end{array}$ \\
\hline$T^{*}$ & Characteristic temperature parameter \\
\hline$p^{\prime}, p$ & Empirical constants \\
\hline Eq. & Equation \\
\hline
\end{tabular}

\section{Greek Letters}

$\omega_{1} \quad$ Mass fractions of liquid 


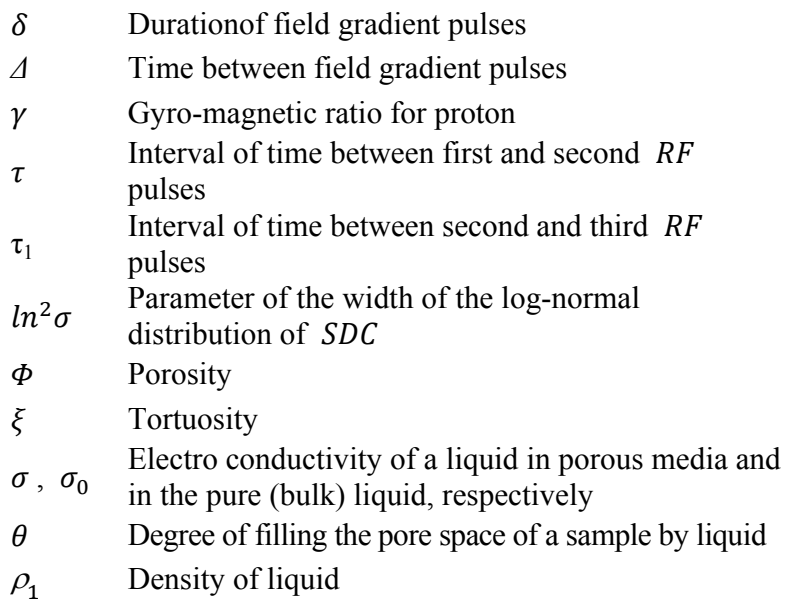

\section{Introduction}

The problem of extracting hydrocarbons from natural reservoirs is one of the important tasks for oil-producing companies $[1,2]$. Clearly to solve it needs information about the behavior of hydrocarbons at the molecular level in the oil-saturated reservoirs, which are natural porousmedia. This information may be obtained by studying the self-diffusion process [3, 4]. For example, in the systems oil - a model porous medium [5]. Thus, from scientific and practical point of view the study of liquid self-diffusion through a porous medium is relevant.

The purpose of this work is the study of self-diffusion of natural hydrocarbon liquids (crude oil) through the clay porous media and the determination of their molecular state in this media.

\section{Experiment}

\subsection{Materials}

Oil samples taken from different oilfields of the Republic Tatarstan (Russia) were used as a natural liquids. All fluids were divided into two groups: light oil (LO) and heavy or highly viscous oil ( $H O)$. Designations and properties of the samples were shown in Table 1.

The porous medium used was a powdered kaolinite compound extracted from the Glukhovetskoe (Ukraine) deposit. The chemical formula for this clay mineral was written as $(\mathrm{OH})_{8} \mathrm{Si}_{4} \mathrm{Al}_{4} \mathrm{O}_{10}$. Kaolinite has sufficient developed specific surface area $S_{1}$, about $9.9 \mathrm{~m}^{2} / \mathrm{g}$, determined by simplified method of air adsorption [6]. The oil - clay samples were prepared the following way: the kaolinite powder was previously sieved through a sieve with the size of openings diameter $\sim 0.063 \mathrm{~mm}$. Then required amount of oil (as defined by the concentration of the sample) was dissolved in a chemically pure grade toluene, which was used as solvent for all investigated samples. Obtained solution was thoroughly mixed with the required amount of sieved clay, and the solvent was evaporated to constant weight.

The prepared sample was placed in a glass test-tube with an outer diameter of $\sim 7 \mathrm{~mm}$ (according to size of the device sensor) and the tube was sealed. The liquid mass fractions $\left(\omega_{1}\right)$ in the studied samples have ranged in the interval from $\omega_{1}=0.05$ to $\omega_{1}=$ 1.00 .

\subsection{Method of Measurement}

Translational molecular mobility - namely the SD (Self-diffusion) process in the system the liquid-porous media was studied by PFG NMR method [3-5]. All diffusive measurements were performed on the local NMR spectrometer at the Department Physics of Almetyevsk State Oil Institute (Tatarstan, Russia). This device operates at a proton

Table 1 Characteristics of the studied oils.

\begin{tabular}{lll}
\hline Designations & Deposit & Comment \\
\hline$L O-1$ & Shijskoe (oil well № 53) & Standard (crude) oil \\
$L O-2$ & Shijskoe (oil well № 552) & Standard (crude) oil \\
$L O-3$ & Romashkinskoe (oil well № 10321) & Standard (crude) oil \\
$H O-A$ & Ashalchinskoe (oil well № 195) & High temperature fraction of standard oil with $T_{\text {boiling }}>623 \mathrm{~K}$ \\
$H O-M$ & Mordovo-Karmalskoe (oil well № 328) & Extract from the oil-saturated rock core \\
\hline
\end{tabular}




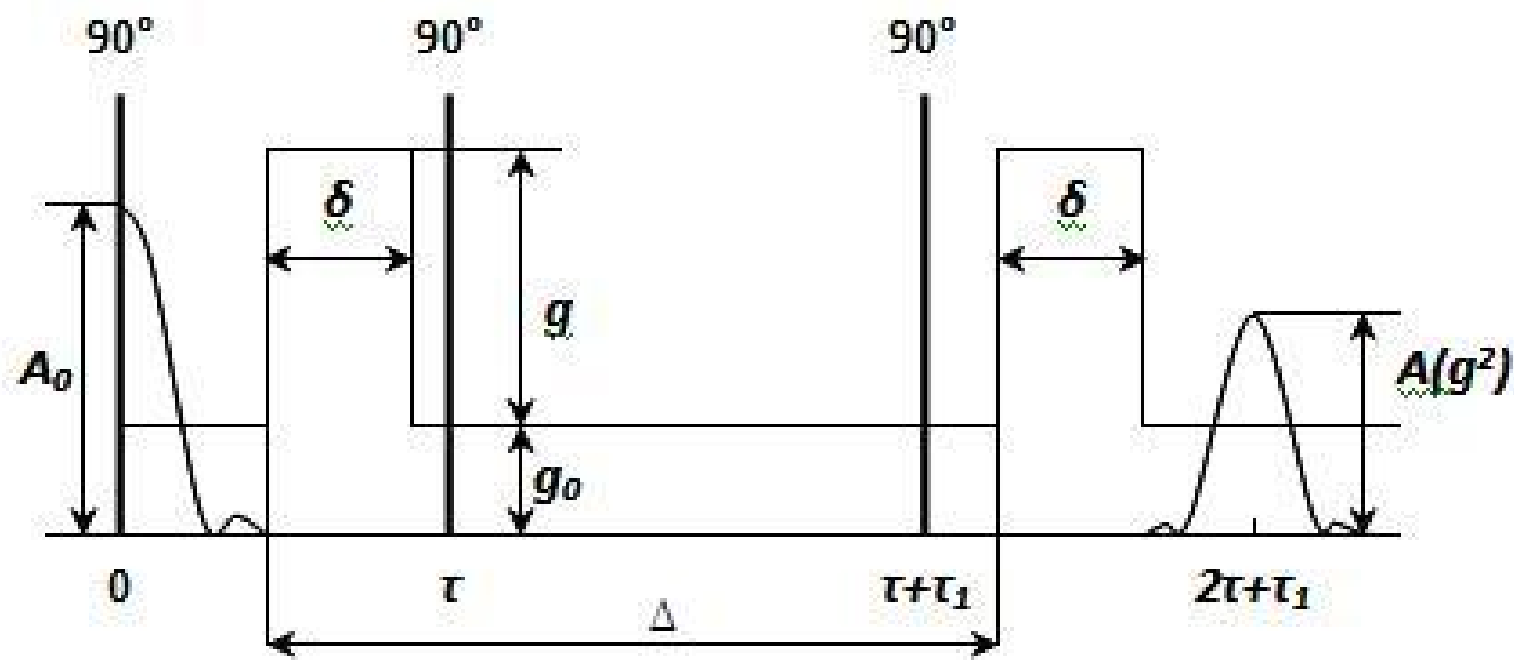

Fig. 1 Three-pulse stimulated spin echo sequence for pulsed field gradient self-diffusion measurements [4]: $90^{\circ}$ - pulses are $R F$ frequency; $g$ and $\delta$ are, respectively, an amplitude and a duration of field gradient pulses, $\Delta-$ is the time between them; $g_{0}$ - is a constant gradient; $\tau$-interval of time between first and second $R F$ pulses; $\tau$-interval of time between one and second $R F$ pulses; $\tau_{1}-$ interval of time between second and third $R F$ pulses.

resonance frequency $64 \mathrm{MHz}$ and provides a maximum value of $40(\mathrm{~T} / \mathrm{m})$ for the intensity of $P F G-g$.

The experiments used the standard stimulated echo pulse sequence (Fig. 1). The observation time of the diffusion process $t_{d}=(\Delta-\delta / 3) \quad(\delta-$ is the durationof field gradient pulses and $\Delta$ is a time between them) was varied within the range $(5 \div$ 24) $m s$.

In case of isotropic and unrestricted diffusion, the intensity of stimulated spin echo amplitude is given by the following expression $[3,4]$ :

$$
\begin{gathered}
A_{\text {stim }}=\left(\frac{A_{0}}{2}\right) \exp \left(-\frac{2 \tau}{T_{2}}-\frac{\tau}{T_{1}}\right) \\
\exp \left(-\gamma^{2} \delta^{2} g^{2} t_{d} D\right) d D
\end{gathered}
$$

where, $A_{0}$ denotes the initial amplitude of the free induction signal after applying the first $90^{\circ}-$ radiofrequency $(R F)$ pulse (Fig. 1), $T_{1}$ and $T_{2}$ are the longitudinal and transverse relaxation times respectively, $\gamma$ is a gyro-magnetic ratio for proton, $D$ is a self-diffusion coefficient $(S D C)$.

In our work the attenuation of spin echo amplitude due to the self-diffusion process (diffusion attenuations $(D A))$ has been registered at the fixed values $\delta$ and $t_{d}[4,5]$. This method is the most suitable, as the time interval between $R F$ pulses remains constant. Consequently the contribution of the constant relaxation decay term $\left(A_{0} / 2\right) \exp \left(-2 \tau / T_{2}-\tau_{1} / T_{1}\right)$ can be excluded from consideration in the processing of the experimental curves. In accordance Eq. (1), the value of the measured self-diffusion coefficient was determined from the slope of dependence $\ln A_{\text {stim }}=f\left(g^{2}\right)$. PFG NMR parameters were calibrated using a standard liquid, namely distillated water, which has an $S D C$ of $(2.7 \pm 0.1) \cdot 10^{-9}\left(\mathrm{~m}^{2} / \mathrm{s}\right)$ at $T=303 \mathrm{~K}$.

\section{Results and Discussion}

An example of attenuations of the spin echo amplitude due to the self-diffusion process $(D A)$ measured in pure (volume) oil (curve 1) and for some systems oil - clay (curves 2 and 3) is depicted in Fig. 2.

The experimental dependences $\lg A\left(g^{2}\right)$ have a complex, not the exponential form. According to coordinates indicated in Fig. 2, the $D A$ in all other cases had the similar shape which differed from one shape to another only by the degree of deviation from the straight line. Formally, each of the non-exponential 


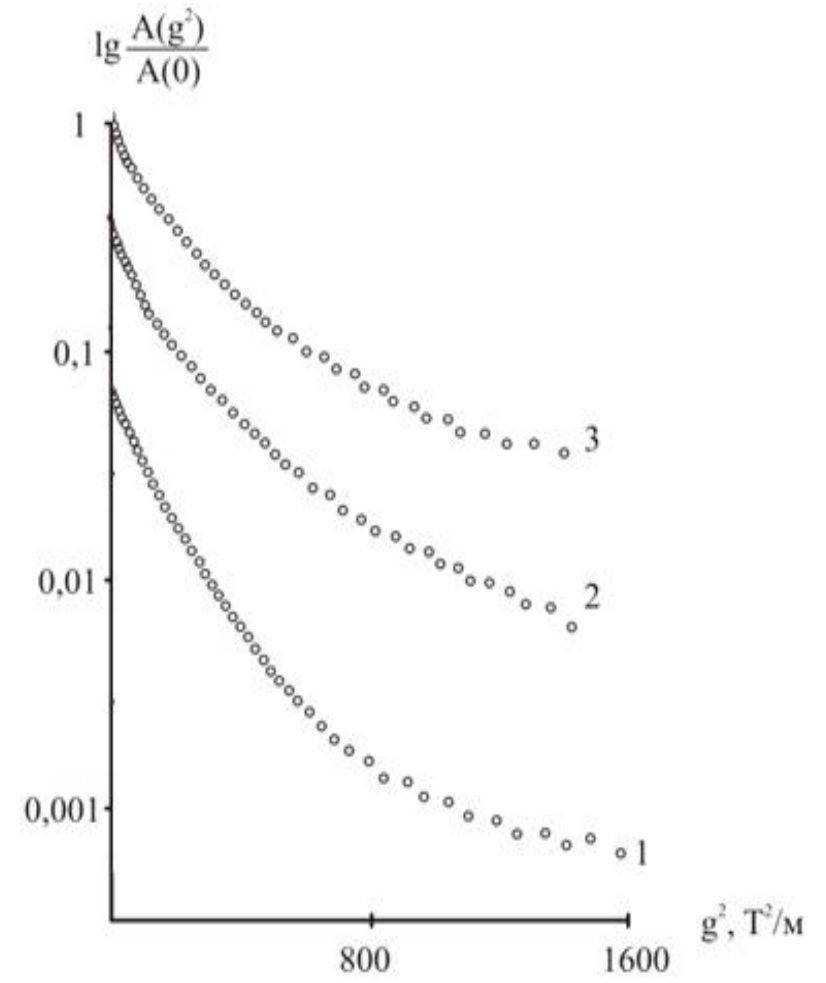

Fig. 2 Curves of diffusion attenuation in $(\mathrm{LO}-3)$ kaolinite systems for samples with $\omega_{1}=100 \%$ (curve 1); $\omega_{1}=21.9 \%$ (curve 2) and $\omega_{1}=14.2 \%$ (curve 3) taken at $\mathrm{T}=303 \mathrm{~K}$ are shown. In all cases $t_{d}=13 \mathrm{~ms}, \delta=$ $0.25 \mathrm{~ms}, \tau=2.0 \mathrm{~ms}$. Positions curves relative to the y-axis is arbitrary.

curves can be analytically represented as [4]:

$$
\frac{A\left(g^{2}\right)}{A(0)}=\int_{0}^{\infty} P(D) \exp \left(-\gamma^{2} \delta^{2} g^{2} t_{d} D\right) d D
$$

where, $P(D)$ is the share of the resonating nuclei in a sample with a value for $S D C$ in interval from $D$ to $(D+d D)$.

The self-diffusion process which is characterized by a non-exponential diffusion attenuations $A\left(g^{2}\right)$ can be described by an effective or average value for the entire sample volume self-diffusion coefficient $D_{e f f}$ [4]:

$$
D_{e f f}=\int_{0}^{\infty} P(D) D d D
$$

Then the experimental value of $D_{e f f}$ is determined from the initial slope of the curve $A\left(g^{2}\right)$, i.e.:

$$
D_{e f f}=-\lim _{g \rightarrow 0}\left\{\frac{1}{\gamma^{2} \delta^{2} t_{d}} \cdot \frac{\partial\left[\ln A\left(g^{2}\right)\right]}{\partial\left(g^{2}\right)}\right\}
$$

In many cases the function $P(D)$ is approximated by the log-normal distribution function of the $S D C$ [4]:

$$
P(D)=\frac{1}{\sqrt{2 \pi \ln ^{2} \sigma}} \exp \left[-\frac{\ln ^{2}\left(\frac{D}{D_{n}}\right)}{2 \ln ^{2} \sigma}\right]
$$

where, $D_{0}$ is the most probable value of the $S D C$ and $\ln ^{2} \sigma$ is the parameter of width of the log-normal distribution of $S D C$, which characterizes the value deviation of diffusion attenuation from exponential view. Parameters $D_{0}$ and $\ln ^{2} \sigma$ of the Eq. (5), were chosen by fitting expression (2) to the measured values of $A\left(g^{2}\right)$. The values of these parameters in some oil-clay systems with different $\omega_{1}$ are presented in Table 2. We can see that parameter $\ln ^{2} \sigma$ increases when the liquid fraction in the specimen decreases; thus, it is inversely proportional. Moreover, broadening the $S D C$ spectrum for light oil (sample $L O-3$, for example) becomes very significant.

The dependences of average self-diffusion coefficients on the concentration of liquid in the samples are shown on Fig. 3. All experimental curves can be divided into two groups. The first group includes the curves describing the self-diffusion in systems heavyoil-kaolinite (Fig. 3, curves 4-6). They have an "ordinary" form, indicating a decrease in translational molecular mobility of the liquid with the decrease of its share $\omega_{1}$ in the porous medium.

The second group includes dependences $D_{\text {eff }}\left(\omega_{1}\right)$ describing the diffusion process in systems of light oil - kaolinite (Fig. 3, curves 1-3). These curves have the anomalous view. Measured $S D C$ is reduced with decreasing $\omega_{1}$ from $\omega_{1}=1.00$ to a certain value

Table 2 Parameter values of the width log-normal distribution $\ln ^{2} \sigma$ in oil-clay systems.

\begin{tabular}{lllllll}
\hline \multirow{2}{*}{ Designations of samples } & \multirow{2}{*}{ Temperature, $\mathbf{T}(\mathbf{K})$} & \multicolumn{5}{c}{ Values $\boldsymbol{l n}^{2} \boldsymbol{\sigma}$} \\
\cline { 3 - 7 } & & $\boldsymbol{\omega}_{\mathbf{1}}=\mathbf{1 . 0 0}$ & $\boldsymbol{\omega}_{\mathbf{1}}=\mathbf{0 . 2 2}$ & $\boldsymbol{\omega}_{\mathbf{1}}=\mathbf{0 . 1 5}$ & $\boldsymbol{\omega}_{\mathbf{1}}=\mathbf{0 . 1 0}$ & $\boldsymbol{\omega}_{\mathbf{1}}=\mathbf{0 . 0 5}$ \\
\hline$L O-3$ & 303 & 0.44 & 1.26 & 1.94 & 3.11 & 4.44 \\
$H O-M$ & 333 & 0.57 & 0.82 & 0.92 & - & - \\
$H O-A$ & 423 & 0.41 & 0.47 & 0.80 & 0.88 & - \\
\hline
\end{tabular}




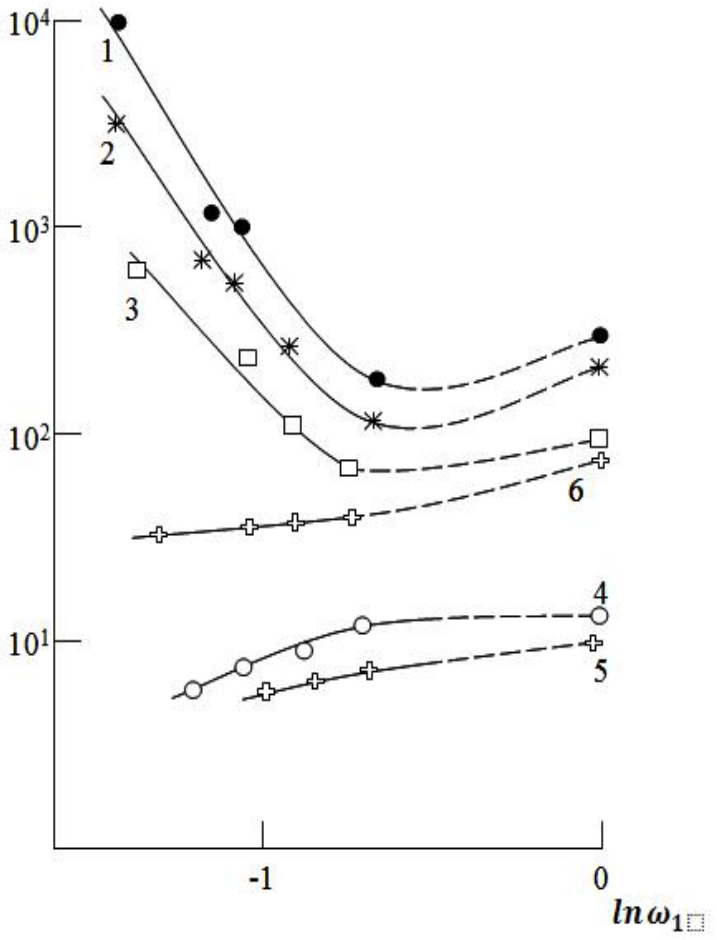

Fig. 3 The concentration dependences of the average $S D C$ in systems:

( $L O-1)$ - kaolinite at temperature $T=303 K$ (curve 1 );

( $L O-2)$ - kaolinite at $T=303 K$ (curve 2);

( $L O-3$ )- kaolinite at $T=303 \mathrm{~K}$ (curve 3); as well as

$(H O-M)$ - kaolinite at $T=333 K$ (curve 4);

$(H O-A)$ - kaolinite at $T=363 K$ (curve 5);

$(H O-A)$ - kaolinite at $T=433 K$ (curve 6).

$\omega_{1}=\omega_{1}^{*}$ (dotted part the curves). However, further reduction of the fraction of liquid in the sample in range $\omega_{1}<\omega_{1}^{*}$ leads to a sharp increase in $S D C$ values which can take values much larger than $S D C$ of pure (volumetric) light oil (Fig. 3, curves 1-3). It should be noted that the appearance the ascending (anomalous) plot of the curve $D_{\text {eff }}\left(\omega_{1}\right)$ can be possible only under partial filling with liquid in the porous space of sample, i.e. when part of the total volume of pores is left free from liquid. Also we have to note that dependence was not found for the measured $D_{e f f}$ from diffusion time $t_{d}$ in interval from $5 \mathrm{~ms}$ to $24 \mathrm{~ms}$. Independence of the measured $S D C$ from diffusion time can be explained using the Einstein-Smoluchowski equation [4]:

$$
\left\langle r^{2}\right\rangle=6\langle D\rangle t_{d}
$$

This equation gives us the possibility to estimate the mean-square values of runs $\left\langle r^{2}\right\rangle$ of diffusing molecules, the minimum of which in our experiments is about 0.4 microns. In its turn, the average pore size of used kaolinite $\langle d\rangle$, can be estimated [7] by the formula:

$$
\langle d\rangle=4 V_{0} / S_{1}
$$

where, $V_{0}$ is specific pore volume and $S_{1}$ is specific surface area. Since for used kaolinite the $V_{0} \simeq$ $0.36 \mathrm{~cm}^{3} / \mathrm{g}$ [8] and $S_{1} \simeq 9,9 \mathrm{~m}^{2} / \mathrm{g}$; therefore, $\langle d\rangle \sim 0.145$ microns, which is significantly less than the minimum value of experimentally registered runs of diffusing molecules, indicating that in our experiments even the smallest $t_{d}=5 \mathrm{~ms}$ diffusing molecules had time to collide repeatedly with obstacles (the hard particles of kaolinite). Therefore, the measured $D_{\text {eff }}$ cannot be dependent from diffusion time and are regarded as an effective $S D C$ acting as the characteristic parameter of the oil-clay systems studied in our case.

Consider the dependences $D_{e f f}$ on content of liquid $\omega_{1}$ in a porous medium kaolinite. An accurate theory has not previously been well developed to describe the self-diffusion of molecules of a liquid in a porous medium. However, it may be possible to compare the self-diffusion process with the conductivity of liquid as an electric current in the electrolyte associated with the apparent mass transfer [9]. Self-diffusion coefficients $D$ and conductivity $\sigma$ are related by known Einstein equation [9], which when applied to the electrolyte in the porous medium with a porosity of $\phi=V_{0} / V_{T}$ leads to the expression:

$$
\frac{\sigma}{\sigma_{0}}=\Phi \cdot \frac{D}{D_{0}} \cdot \frac{V}{V_{0}}
$$

where, $V_{T}$ is total specific volume of the porous sample, $V$ is the liquid's volume in it and $V_{0}$ - full pore volume. Physical values $\sigma$ and $D$ are used as the transport characteristics of a liquid in porous media, in its turn $\sigma_{0}$ and $D_{0}$ are the same parameters for the pure (bulk) liquid. 
We should note that Eq. (8) is able to take into account only the effect of steric constraints on the transport processes due to the increase in the tortuosity $\xi$ of the diffusion motion of the molecule in a porous medium with respect to movement in a pure liquid. For example, if $l$ is the distance of molecule between two points in a porous medium and $l_{0}$ is the distance between them in a pure liquid, then $\xi=l / l_{0}$.

It is known that the fraction of liquid in the total pore volume is called, by degree of filling $\theta=V / V_{0}$. Notice, that the ratio of $\sigma / \sigma_{0}$ can be associated with the values of $\theta$ and $\Phi$ by Archie's law [9]:

$$
\frac{\sigma}{\sigma_{0}}=\Phi^{p^{\prime}}(\theta)^{p}
$$

In turn the parameter $\theta$ can be find as $\theta=$ $\omega_{1} /\left(1-\omega_{1}\right) \rho_{1} V_{0}$, where $\rho_{1}$ is the density of liquid. Then, the theoretical Eq. (8) can be represented as:

$$
\frac{\mathrm{D}}{\mathrm{D}_{0}}=\Phi^{p^{\prime}-1}\left(\frac{\omega_{1}}{1-w_{1}} \cdot \frac{1}{\rho_{1} V_{0}}\right)^{P-1}
$$

The Eq. (10) allows us to describe translational molecular mobility of the fluid in a porous medium, depending on its concentration $\omega_{1}$. Parameters $p^{\prime}$ and $p$ in Eqs. (9) and (10) are empirical constants. The formal differences between the $p^{\prime}$ and $p$ is allowed because the topology of the liquid which is completely filling pore space may be different from the topology of the fluid filling this space partially.

Using the complex natural objects (oil and clay) in our work creates great difficulties in carrying out accurate quantitative calculations of $S D C$ in order to compare them with experimental data. Moreover, the force interaction between diffusing molecules and the surface of the solid phase have not been taken into account in Eqs. (8) and (10), which must take place in such systems [10]. However, following the qualitative analysis, the experimental results are in agreement with Eq. (10). In fact, according to Eq. (10), the measured $S D C$ must be reduced by decreasing the share of liquid in the system, specifically by the results observed in our experiments (Fig. 3, curves 4-6 and curves 1-3 in area $\omega_{1}>\omega_{1}^{*}$ ).

It is interesting to detect the reasons for the appearance anomalous curve of dependence $D_{\text {eff }}\left(\omega_{1}\right)$ when $\omega_{1}<\omega_{1}^{*}$, that is, under the partial filling by fluid the pore space. This result indicates upon experimental confirmation the effect of enhanced self-diffusion under certain conditions, specifically, that the measured $S D C$ of liquid concluded in porous medium can exceed the $S D C$ in pure (bulk) fluid.

The fact that the experimentally determined $S D C$ liquid in a porous medium may be greater than that of the pure (bulk) fluid, was first discovered by Boss and Stejskal [11] in the study of self-diffusion the water in hydrated vermiculite. Karger et al. [12] later observed a similar effect on the water in $N a Y$ - zeolite. In the first case, the authors used the hypothesis formation of a vapor phase on crystal faces to interpret the unusual result; in the latter, the result remained unexplained.

Abnormal curves $D_{e f f}\left(\omega_{1}\right)$ were later found by D'Orazio et al. [9] in the study of self-diffusion of water in porous glass, and by us [13] in the study of self-diffusion of some alkanes and water in the clay. An explanation of results in both cases were practically identical and done independently from each other.

The state with anomalously enhanced self-diffusion in our works has been described as "gas-like" state. This given state can be realized only under certain conditions. First, the porous medium should be only partially filled with liquid; the oil and its saturated vapor coexist together in porous space of a sample. Second, the medium should be of sufficiently high specific surface area $S_{1}$. The fluid must also has a sufficiently low boiling point, and the measurements should be performed at sufficiently high temperatures.

The physical essence of the gas-like state is that in the listed higher conditions between the liquid and its gaseous component is realized fast (in terms of NMR) molecular exchange, which should involve all the diffusing molecules. Moreover, at fast molecular 
exchange between the coexisting phases should be performed inequality [4]:

$$
t^{L}, t^{G} \ll t_{d}
$$

where, $t^{L}$ and $t^{G}$ are the "lifetime" of the molecule in the liquid and gas phases, respectively. In that case, the average value of the measured effective $S D C D_{\text {gaslike }}$ can be written [11] as:

$$
\begin{aligned}
D_{\text {gaslike }} & =P^{L} D^{L}+P^{G} D^{G}, \\
\boldsymbol{P}^{L} & +\boldsymbol{P}^{G}=\mathbf{1}
\end{aligned}
$$

where, $P^{L}$ and $P^{G}$ are the relative fraction of molecules in a typical liquid and gaseous states; $D^{L}$ and $D^{G}$ is $S D C$ values that characterize these states, respectively.

Contribution to the measured $D_{\text {eff }}\left(\omega_{1}\right)$ from the vapor phase will be observed in the event that both terms in Eq. (8) will be comparable. For example, for the system $(L O-3)$ kaolinite at $T=303 \mathrm{~K}$ it will be possible when the $P^{G}$ has value of $\sim 10^{-5}$, as the $S D C$ for the gas molecules under normal conditions is usually $D^{G} \sim 10^{-5} \mathrm{~m}^{2} / \mathrm{s} \quad$ [14]. Obviously, the higher the value of $P^{G}$ is, the higher the probability of the experimental observation of enhanced self-diffusion has. This means that, all other conditions being equal, the liquid must be "readily volatile", i.e. to have a sufficiently low boiling point.

It is well known [2] that the oil is characterized by a wide range of hydrocarbon compounds, from the lightest paraffinic fractions to heavy (high-molecular) resins and asphaltenes. The ordinary (light) oil contains a sufficiently large proportion of volatile components. Therefore the presence this type of oil in porous space of a sample may implement the appearance the gas-like state, which we directly observed for the systems $\mathrm{LO}-$ kaolinite when $\omega_{1}<\omega_{1}^{*}$ (Fig. 3, curves 1-3).

As for to the heavy oil, it should be noted that they are practically devoid of low molecular weight volatile components that can pass into the gas phase under normal conditions [2]. This may be explained the absence of anomalies in the depending $D_{\text {eff }}\left(\omega_{1}\right)$ for systems: $(H O-M)-$ kaolinite (Fig. 3, curve 4) and $(\mathrm{HO}-\mathrm{A})-$ kaolinite (Fig. 3, curves 5 and 6). Furthermore, measurements of the $S D C$ in these systems may only be performed at much higher temperatures than normal temperature (Fig. 3, curves 4-6). These results may be explained as follows. Heavy oil contains a high proportion of high-molecular components, especially such as resins and asphaltenes which under normal $(\sim 303 K)$ temperatures are in the solid state [15]. Therefore, heavy oils should be characterized by a very short $\left(\sim 10^{-5} \div 10^{-4}\right) s$ nuclear magnetic relaxation times, which under these conditions do not permit us to measure $S D C$ in such samples by $N M R P F G$ method [4].

Transition of heavy oil to the liquid state requires considerable increase the temperature to a value $T=T^{*}$ at which translational movement of high-moleculare components may be detected by NMR PFG method. These temperatures for different systems have been found experimentally by an amplitude of the spin echo signal. On the other hand, the temperature $T^{*}$ may serve as a characteristic parameter, with its own certain value for each system. For example, the system $(\mathrm{HO}-\mathrm{A})$ is characterized by the value $T^{*} \sim 423 \mathrm{~K}$, and the system $(H O-M)$ by the value $T^{*} \sim 363 \mathrm{~K}$. The knowledge of these temperatures may be useful in practice for thermal stimulation methods of bitumen and heavy oil.

In regards to the shape of the diffusion attenuation, different reasons lead to non-exponential form of these curves. These include the molecular weight distribution of the diffusing liquid [4], the heterogeneity properties of the porous medium [13] among others. Crude oil is a collection of a wide range of hydrocarbon compounds: paraffins, naphthenes, olefins, aromatics, and also heteroorganic (containing oxygen, nitrogen, sulfur, etc.) components $[15,16]$. Components of oil, with different molecular weight, should be different in their ability to translational molecular movement. Therefore, the total $D A$, which 
characterizes the whole sample will be determined by a combination of contributions from the various components, where each component has its own $S D C$ (Fig. 1, Table 2).

We can see from Table 2 that the width of the distribution $\ln ^{2} \sigma$ increase when the proportion of liquid $\omega_{1}$ in a sample decreases. This is, apparently, the result of the manifestation of the increasing power of the molecular interaction between the components of oil and the surface of solid particles when shared in the latter of the sample which is increased. The value of the $\ln ^{2} \sigma$ parameter in the systems light oil kaolinite increases much more sharply with decreasing $\omega_{1}$, compared to samples with heavy oil (Table 2). This result confirms existence the heterogeneity of properties of the natural kaolinite, which is most manifested when some share of used liquid transforms to the gas - like state [13].

\section{Conclusions}

Thus, the obtained results give us information about the molecular state of oil when this natural liquid is in the porous media. Method PFG NMR is used very successfully for solving such problems. An anomalously enhanced self-diffusion in samples of light oil - kaolinite was detected under specific conditions. The unusual state with enhanced self-diffusion is caused by presence in the free part of the pore space of the sample in the vapor phase of liquid. State of enhanced self-diffusion in this case is explained by presence in the free part of pore volume the vapor phase. Fast molecular exchange between coexisting liquid and gas phases provides fulfillment enhanced self-diffusion in the light oil - kaolinite system.

\section{Acknowledgements}

Author is sincerely grateful to Professor of Physics the Kazan Federal University Alexander Ivanovich Maklakov for participation in the discussion of the materials in this article. Many thanks for his useful advice.

\section{References}

[1] Miloserdova, L. 2007. "Geology, Prospecting and Exploration of Oil and Gas." Edited by "Maks Press", Moscow, 321.

[2] Hunt, D, 1982. "Geochemistry and Geology of Oil and Gas." Edited by "The World". Moscow, 703.

[3] Tanner, J. 1970. "Use of the Stimulated Echo in NMR. Diffusion Studies." J. Chem. Phys. 52 (5): 2523-6.

[4] Maklakov, A., Skirda, V. and Fatkullin, N. 1990. "Self-Diffusion in Polymer Systems, Encyclopedia of Fluid Mechanics." In Polym. Flow Engineering, edited by Cheremisinoff, N. P. Vol. 9. Gulf Publishing Comp., Houston, London, Paris, Zurich, Tokio. Chapter 22, 705-45.

[5] Maklakov, A., Dvoyashkin, N. and Tyurin, V. 2001. "Studing the Fluid-Saturated Porous Media by NMR Technique." Edited by Georesources, Kazan State University. 1: 32-4.

[6] Huziahmetov, R., Khusnutdinov, V., Dvoyashkin, N. and Saifullin, R. 1993. "A Method for Producing Active Magnesium Oxide.” USSR. Patent 1787939 B1(N2).

[7] Dzisko, V., Karnaukhov, A. and Tarasov, D. 1978. "Physico-Chemical Basis of the Synthesis of Oxide Catalysts." Edited by "Science", Novosibirsk, 384.

[8] Ovcharenko, F., "1961 Hydrophilic of Clays and Clay Minerals." Publishing House of the USSR Academy ofSciences, Kiev, 292.

[9] D'Orazio, F., Bhattacharia, S., Halperin W. and Gerhardt R. 1989. "Enhanced Self-Diffusion of Water in Restricted Geometry." Phys. Rev. Lett. 63 (1): 43-6.

[10] Dvoyashkin, N. and Maklakov, A. 1992. "Features of the Self-Diffusion of Liquids in a Medium with Spherical Obstacles." Journal of Colloidal Chemistry 54 (3): 30-7.

[11] Boss, B. and Stejskal, E. 1969. "Restricted, Anisotropic Diffusion and Anisotropic Nuclear Spin Relaxation of Protons in Hydrated Vermiculite Crystals." J. Colloid Interfase Sci. 26 (2): 271-8.

[12] Karger, J., Pfeifer, H., Riedel, E. and Winkler, H. 1973. "Self-Diffusion Measurements of Water Adsorbed in NaY Zeolites by Means of NMR Pulsed Field Gradient Techniques." J. Colloid Interface Sci. 44: 187-8.

[13] Dvoyashkin, N., Skirda, V., Maklakov, A., Belousova, M. and Valiullin R. 1991. "Peculiarities of Self-Diffusion of Alkane Molecules in Kaolinite." Appl. Magn. Reson. 2 (1): 83-91.

[14] Bretschneider, S. 1966. "Properties of Gases and Liquids (Russian Translation)." Edited by "Chemistry", Moscow, Leningrad, 455. 
[15] Sunyaev, Z. 1984. "Chemistry of Oil." Edited by "Chemistry”, Leningrad, 360.
[16] Goon, R. 1989. "Petroleum Bitumen." Edited by "Chemistry", Moscow, 152. 